B. T. Soifer and G. Neugebauer

Palomar Observatory

California Institute of Technology

Pasadena, California 91125

\title{
INTRODUCTION
}

In this paper we review the observed infrared properties of the general classes of active extragalactic nuclei with the purpose of relating the observations to the mechanisms responsible for the emission processes. We will first give a summary of those observations which define the energy distributions and emission line ratios of broad groups of objects. We will intersperse measurements of specific features throughout the discussion that illustrate definite emission mechanisms.

\section{OBSERVATIONAL DATA}

The shapes of the observed continuum energy distributions of the nuclei of active galaxies are well established and have been available in the published literature of the past few years. A summary of the typical energy distributions of extra galactic sources has been given by Rieke and Lebofsky (1979a) (Figure 1), who give extensive references to early work.

Although the general shapes of the energy distributions are given in Figure 1, studies of extensive numbers of objects show significant differences between the spectra of different objects within one class. Figure 2, from Rieke (1978), shows the spectral distributions of a number of Seyfert galaxies out to $10 \mu \mathrm{m}$ from his study of some 50 Seyferts. Although these show the same general increase in flux density into the near infrared, significant differences are seen. In particular, as noted by Neugebauer et al. (1976), the energy distributions of type 2 Seyferts rise relatively more steeply into the infrared than those of type 1 Seyferts; significant exceptions are, however, present. McAlary, McLaren and Crabtree (1979), from a smaller sample, have divided the Seyferts into three groups on the basis of a 1-5 $\mu \mathrm{m}$ study as shown in Figure 3; these correspond to (a) dominance by the stellar continuum, (b) dominance by non-thermal power law component 


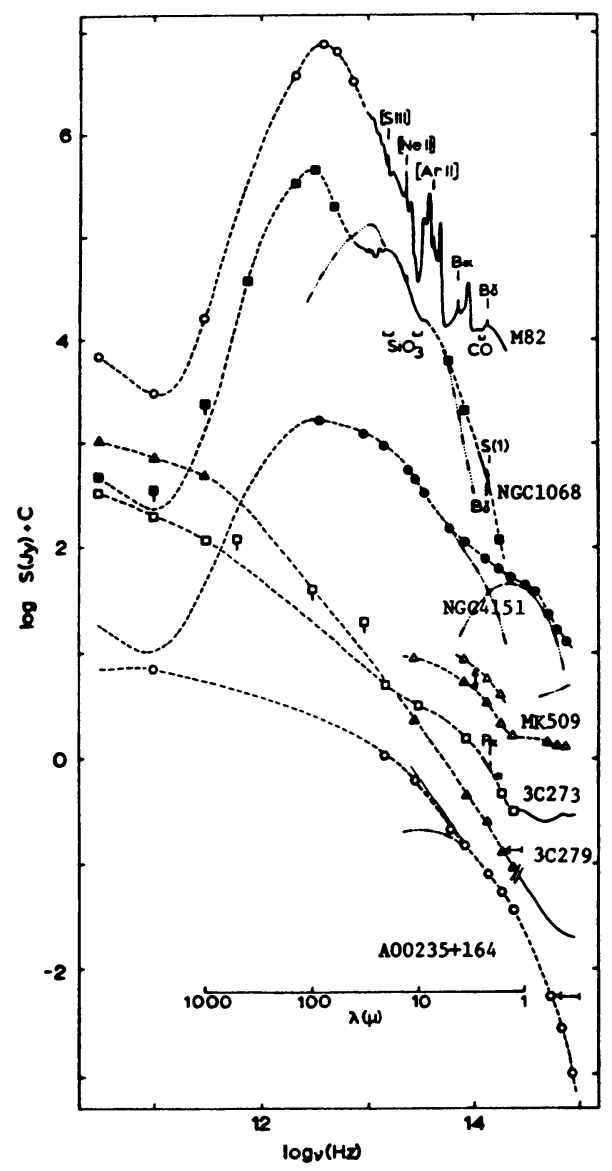

Figure 1. The infrared/optical/radio energy distributions of the major classes of active galactic nuclei (from Rieke and Lebofsky 1979a).

or (c) intermediate cases which contain both components. They find that for $\lambda \sim 2 \mu \mathrm{m}$, type 2 galaxies are largely dominated by stellar continua while the type 1 Seyferts generally show power law spectra in the near infrared. Clearly these results are dependent on the diaphragm used for the observations, since larger diaphragms include more of the stellar radiation, compared to the (nearly) pointlike nucleus. All of the Seyferts which show strong X-ray emission also show evidence of non-stellar emission.

The energy distributions of the quasars are generally typified by that of 3C273 in Figure 1. Detailed near infrared measurements of quasars by Neugebauer et al. (1979) again show significant differences as is clear from Figure $4 a$ and $b$ which show the near infrared energy distributions of low and high redshift quasars. There is no systematic 
difference between the near infrared energy distributions of radio quiet and radio loud quasars. On the other hand, measurements of quasars at $1 \mathrm{~mm}$ correlate extremely closely with the centimeter radio observations (Ennis 1980). The differences between radio quiet and radio loud quasars must therefore show up at about iüu $\mu \mathrm{m}$.

The colors of a "typical" quasar from $2 \mu \mathrm{m}$ to $0.5 \mu \mathrm{m}$ can be generated from the observed $[1.6 \mu \mathrm{m}]-[2.2 \mu \mathrm{m}]$ colors when plotted as a function of red shift. The Caltech data on about 110 quasars are shown in Figure 5, in which the type of survey by which the quasars were found is shown qualitatively. It is seen that within the present sample quasars have similar colors as a function of redshift, independently of how they were discovered, except, perhaps, at the lowest redshifts where those quasars selected from radio surveys have higher $\mathrm{H}-\mathrm{K}$ values than those found from optical and $\mathrm{X}$-ray surveys. From the histograms it appears that, although there is a spread of $\pm 0.5 \mathrm{mag}$ in quasar $\mathrm{H}-\mathrm{K}$ colors, the energy distribution of $3 \mathrm{C} 273$ is "typical" of quasars at all redshifts. The results of Hyland and Allen presented in this conference agree qualitatively with those of Figure 5.

The continua of the final category of violent extragalactic objects, BL Lac objects, are characterized by a much smoother shape than either the Seyferts or quasars. Observations of their energy distributions are difficult because of their rapid variability which is apparently correlated between the visible and infrared. Figure 6 shows the energy distribution of several BL Lac objects from the visual to radio

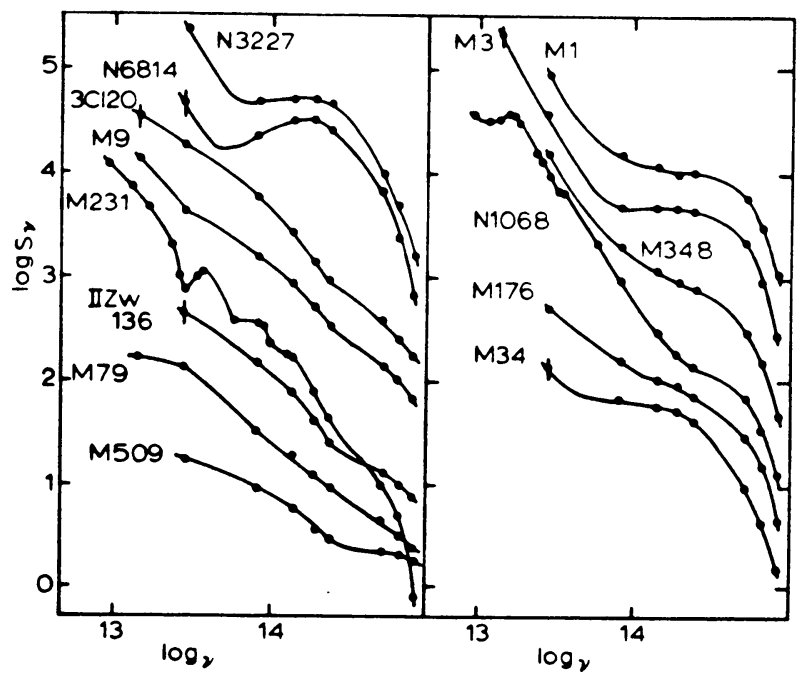

Figure 2. The infrared/optical energy distributions of Seyfert nuclei (from Rieke, 1978). 


\section{OBSERVATIONS OF SEYFERT GALAXIES}
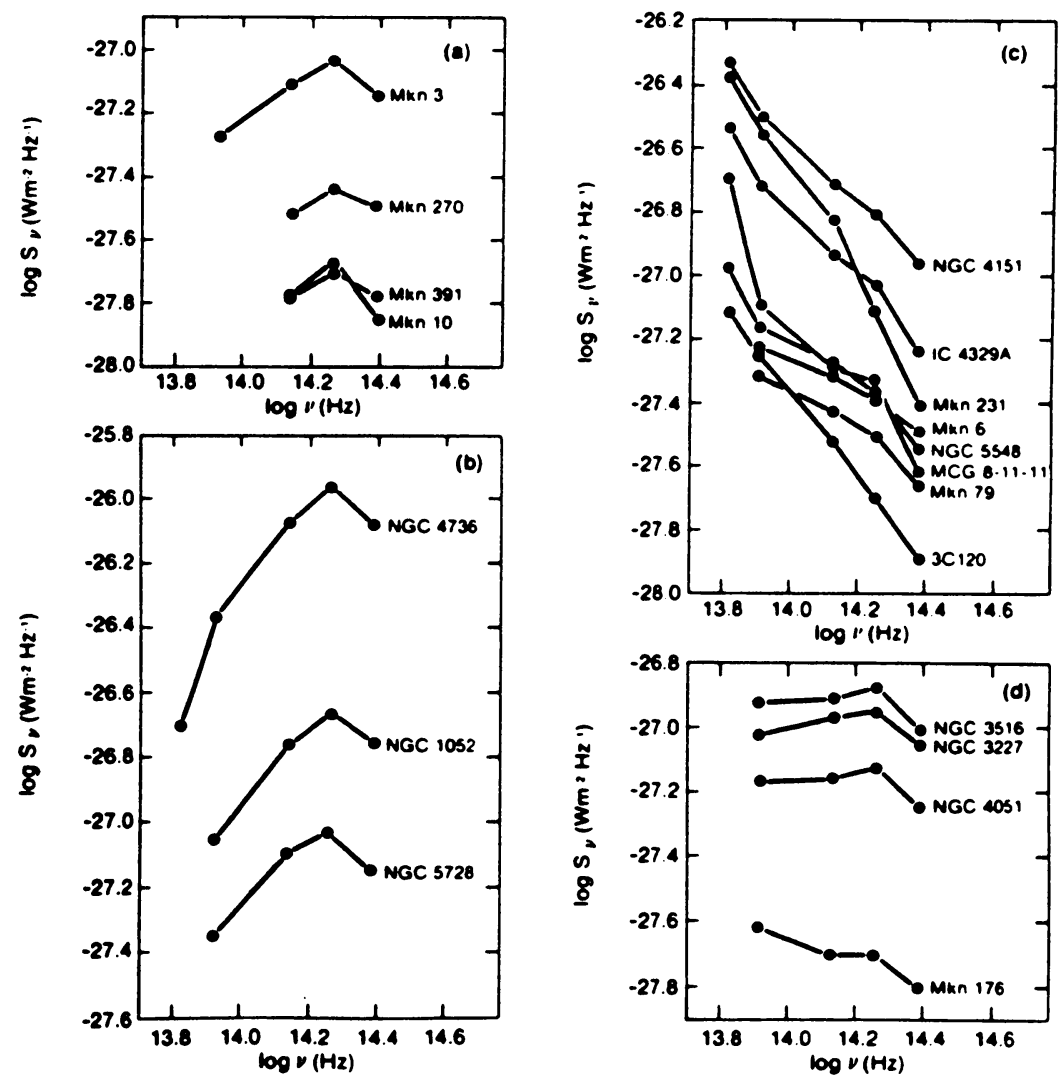

Figure 3. The near infrared energy distributions of Seyfert and emission line galaxies (from McAlary, et al.1979). Box (a) shows the Seyferts with stellar like energy distributions; (b) shows the energy distributions of emission line galaxies; (c) shows the Seyferts with power law spectra; and (d) shows Seyferts with continua intermediate between (a) and (c).

wavelengths as obtained by O'Dell et al. (1978). The apparently smooth distribution is typical of BL Lac itself and at least several of the brighter BL Lac objects.

Recently Rieke, Lebofsky and Kinman (1979) have observed the near infrared radiation from a number of flat-spectrum radio sources in previously unidentified fields; the fluxes are shown in Figure 7 . These objects have far steeper optical-infrared continuum distributions $\left(F_{\nu} \alpha \nu^{-3}\right.$ ) than any other known quasars or BL Lac objects (3C 68.1 comes closest to matching the slope of these sources and has a spectral index $\alpha \sim 2.1)$. Rieke et al. noted the large variability of several of 
these objects and placed them in the class of the optically violently variable quasars. Recently Aaronson and Boroson (1980) have reported visual spectroscopy of one of these objects, $0406+121$, and Soifer, Neugebauer and Matthews have obtained near infrared spectra of two of these objects $(1413+135$ and $0406+12)$. None of the spectroscopic work indicates any evidence for emission lines; this, plus the large variability, suggests that these objects form the extreme red end of the BL Lac class of objects. Another interesting property of these objects is the significant change in continuum slope as a function of brightness illustrated in Figure 8 .
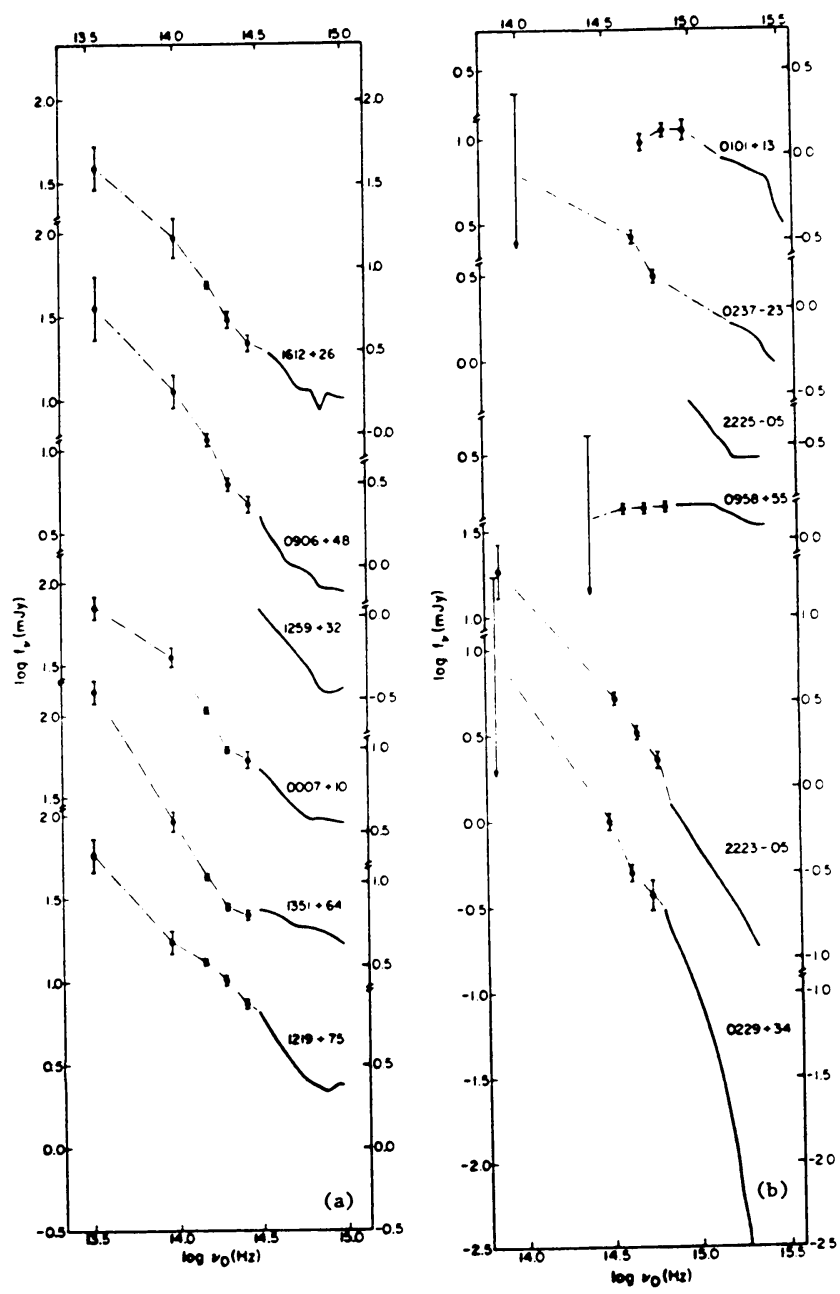

Figure 4. The infrared/optical energy distributions of low (box a) and high (box b) redshift quasars (from Neugebauer, et a1.1979). The fluxes are plotted in the rest frame of the quasars. 


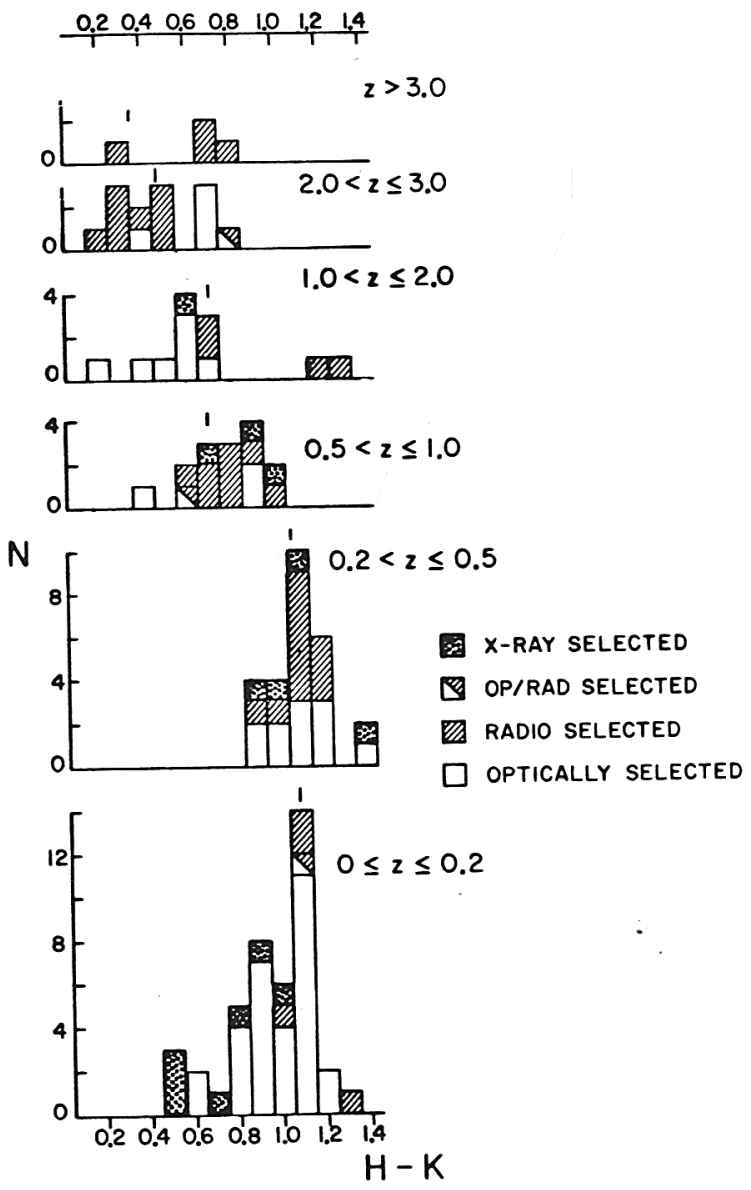

Figure 5. The observed $\mathrm{H}-\mathrm{K}$ colors of quasars plotted in histograms for the redshift ranges shown. Those quasars discovered via optical, radio, and X-ray techniques are shown in separate boxes. For quasars in the appropriate redshift ranges, color corrections have been applied for $H \alpha, H \beta$, and [OIII] emission in the $H$ and/or $K$ bands, taking the mean equivalent widths of these lines from the work of Soifer, et al. (1980a). The vertical tic mark indicates the $\mathrm{H}-\mathrm{K}$ color $3 \mathrm{C} 273$ would appear to have at the mid-range redshift of each histogram. The data on $3 C 273$ are from Neugebauer, et al. (1979).

In recent years, increased emphasis has been placed on observations of the hydrogen emission lines in quasars red-shifted into the near infrared wavelengths. These measurements, which follow the pioneering work of Baldwin (1977), should provide a means of studying the clouds where line formation takes place. The most extensive studies in the infrared have been made by Puetter et al. (1980) and by 

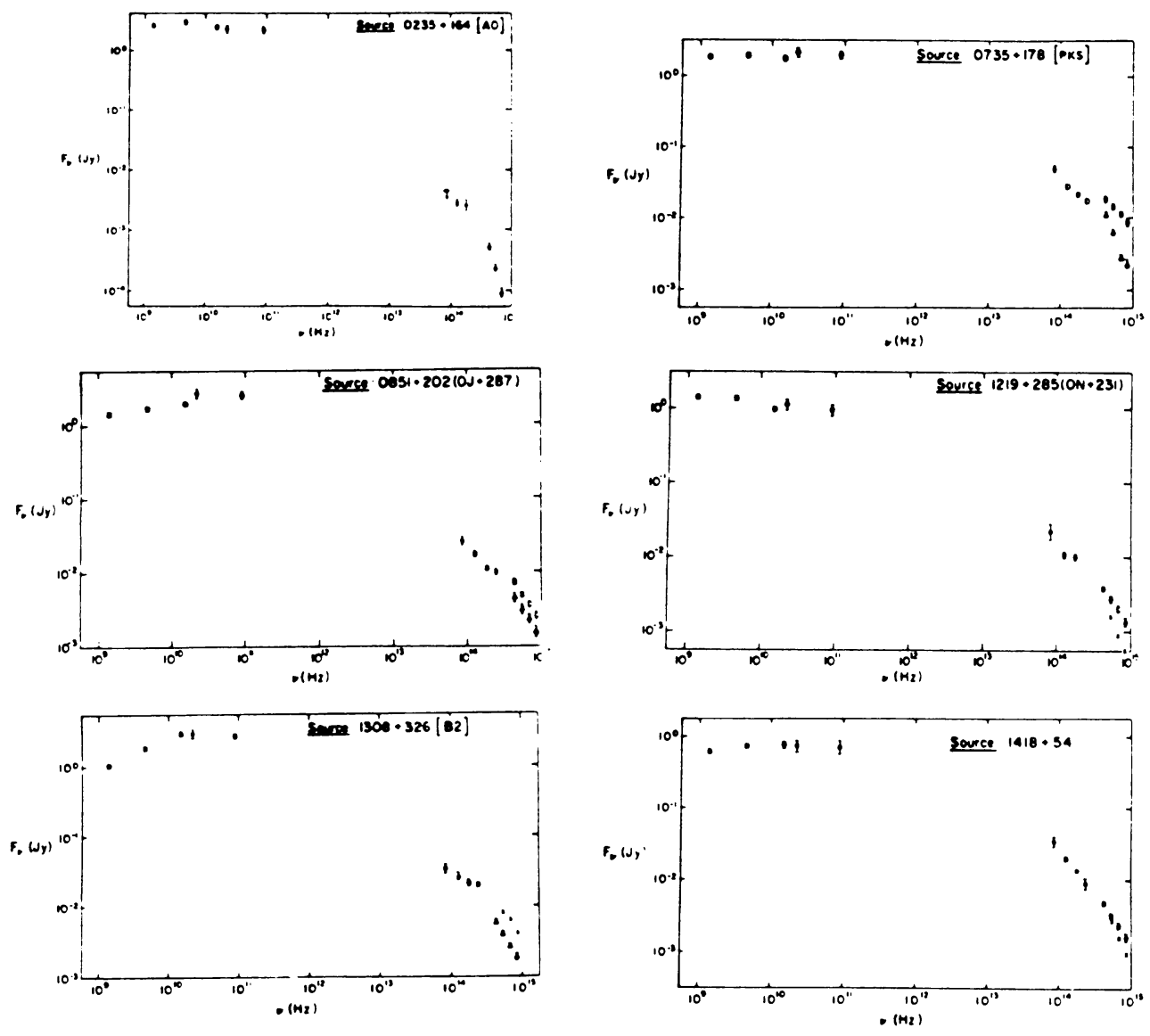

Figure 6. The energy distributions of selected BL Lac objects from the radio through the optical range (from 0'De11, et a1. 1978) All the observations of a given object reported here were obtained within a few months of each other. Even over this time span substantial variability can be seen in the observations of $0735+178,0 J 287,0 \mathrm{~N} 231$, and $1308+32$.

Soifer et al. (1980a); Figure 9 shows their combined data on the $\mathrm{P} \alpha / \mathrm{H} \alpha / \mathrm{H} \beta$ line ratios in quasars. In Figure 10 the $\mathrm{L} \alpha / \mathrm{H} \alpha$ ratio is shown as obtained for both high and low red-shift quasars. Preliminary data on the $\mathrm{P} \alpha / \mathrm{H} \alpha / \mathrm{H} \beta$ ratios for Seyfert galaxies obtained from Mauna Kea (Soifer et al. 1980b) are shown in Figure 11. The differences, which will be discussed below in the context of the dust content of the nuclei, are striking. 


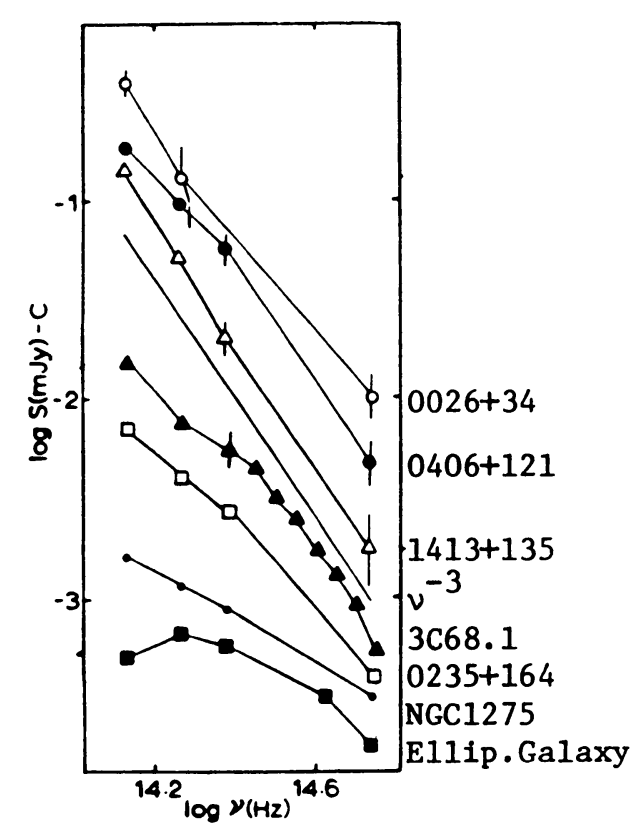

Figure 7. The near infrared/optical energy distributions of a class of radio sources having very steep infrared/optical spectra (from Rieke, Lebofsky, and Kinman, 1979).

\section{DISCUSSION}

The main question that we wish to discuss is what mechanisms are responsible for the observed infrared continuum emission in the Seyferts, BL Lac objects, and quasars. The main mechanisms that have been mentioned as potentially producing the infrared emission are thermal emission by dust in the nuclei, and incoherent synchrotron emission. Other emission mechanisms such as synchrotron self-Compton emission and multiple Compton scattering by dense nonrelativistic plasmas are generally thought to be inapplicable as the infrared emission mechanism (e.g., Jones, et al. 1980, O'De11 1978).

Examples where thermal emission by dust and incoherent synchrotron emission are thought to be the sources of the observed infrared flux can be found in the observations of several classes of active galactic nuclei. The most straightforward example of thermal emission by dust producing the infrared radiation is in the type 2 seyfert nuclei, archetypical of which is the Seyfert galaxy, NGC 1068.

The energy distribution in NGC 1068, as shown in Figure 1, is quite similar to that seen in $M 82$ and galactic $H$ II regions where there is no doubt that the mechanism producing the infrared radiation is 


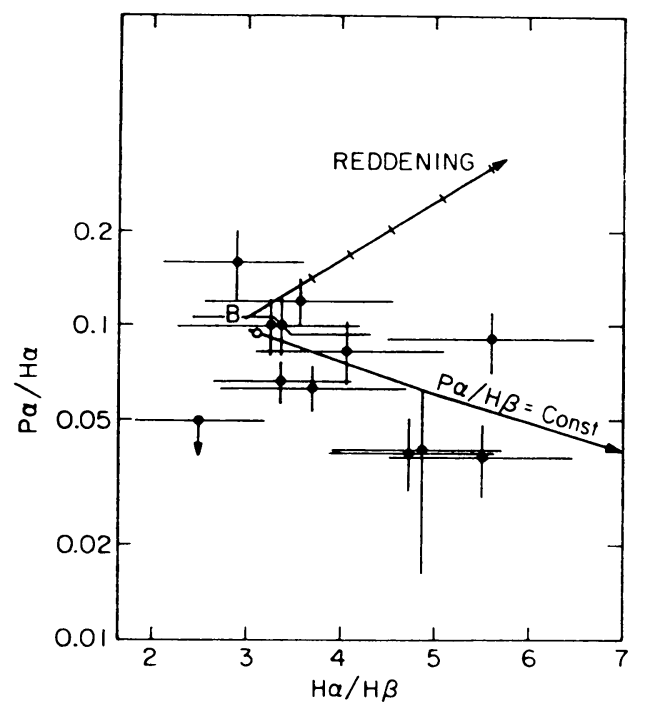

Figure 9. The $\mathrm{P} \alpha / \mathrm{H} \alpha / \mathrm{H} \beta$ line ratios in low redshift quasars. The data are from Soifer, et al. (1980a) and Puetter et al. (1980). The B in the figure indicates the position of the Case $B$ recombination valve. The arrow marked reddening indicates the effect normal interstellar reddening would have on the Case $B$ value. The line marked $\mathrm{P} \alpha / \mathrm{H} \beta=$ Const is the deviation expected from Case $B$ in the case of collisional excitation when the Balmer lines are optically thin.

NGC 1068 in the infrared, the current consensus is that there is no evidence for variability in the infrared emission in NGC 1068. This is therefore consistent with (but does not prove) thermal dust emission models.

In addition to the photometric and angular size measurements, there is spectroscopic evidence for the presence of dust in the nucleus of NGC 1068. It has been known for over a decade (Wampler, 1968) that the S II lines appeared reddened in the nucleus of NGC 1068. Wampler (1971) and Shield and Oke (1975) have also found that the Balmer decrement is large in NGC 1068, possibly (but again, not necessarily) implying the presence of dust in the nucleus of this galaxy. Recently Neugebauer et al. (1980) have shown that the observed strengths of all of the hydrogen emission lines from L $\alpha$ to $\mathrm{P} \alpha$, of He II 1640,4686, and of the S II 4072,10320 lines are consistent with normal line ratios and reddening by $\sim 1.5 \mathrm{mag}$ of visual extinction. Kleinmann, et al. (1976) have obtained a spectrum of NCG 1068 from $8-13 \mu \mathrm{m}$ that shows evidence for silicate absorption, indicating that the $10 \mu \mathrm{m}$ source is viewed through a large column density of obscuring material.

In summary, the observational evidence has pointed to the 
thermal emission by dust. The continuum slope from 1 to $20 \mu \mathrm{m}$, $\alpha \sim-3$, is much steeper than that seen over the same wavelength range in any source where non-thermal mechanisms are thought to dominate; the continuum slope in the visual is sometimes found to be steeper than this, but even in these cases, e.g. $0235+164,3 \mathrm{C} 68.1$, the continuum slope in the infrared is much less than at visual wavelengths.

Perhaps the strongest argument in favor of a thermal dust mode1 in NGC 1068 is the measured size of the infrared source. Becklin et a1. (1973) measured the diameter of the $10 \mu \mathrm{m}$ source to be 1 " or $90 \mathrm{pc}$. This is quite readily explained if the $10 \mu \mathrm{m}$ flux is from dust grains heated by a central ionizing source. It is difficult to understand this observation if the infrared source is somehow related to the non-thermal source observed at optical and ultraviolet wavelengths. Hildebrand et al. (1977) have shown that if the far infrared source is a thermal source, it must be at least $5^{\prime \prime}$ in extent, and Telesco, et al. (1980) have shown that $20 \%$ of the $20 \mu \mathrm{m}$ flux from NGC 1068 is from a region larger than 3" (260 pc) in radius, consistent with the thermal models of the far infrared and submillimeter emission from this object.

Observed variability is often used as an argument against thermal dust emission models, since observed time scales of variability are often much less than that required in such models. Although in the past there has been controversy over the possible variability of

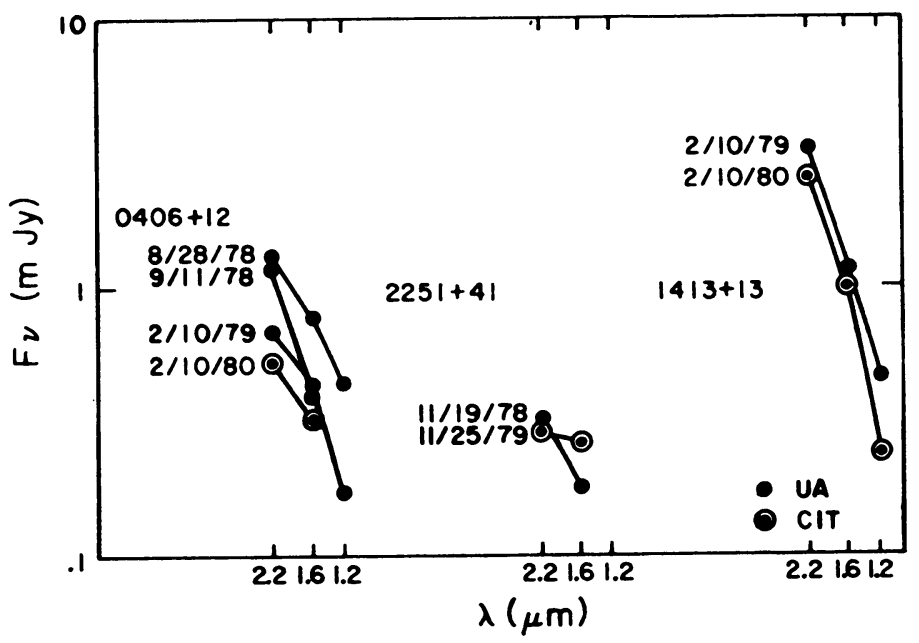

Figure 8. The near infrared energy distributions of three of the objects observed by Rieke, Lebofsky, and Kinman (1979). The energy distributions show significant changes in shape with time. The data noted as UA are from Rieke, et al.; the data noted as CIT are unpublished observations of Soifer, Neugebauer, and Matthews. 
conclusion that the mechanism producing the infrared emission in the archetypical type 2 Seyfert galaxy NGC 1068 is thermal emission by dust. In addition, Rieke's (1978) study of Seyfert galaxies shows that the other type 2 Seyfert galaxies are similar to NGC 1068.

In contrast to the case of NGC 1068, the observational evidence over the last few years suggests that in the BL Lac objects the infrared emission is due to incoherent electron synchrotron emission. The evidence for this is basically guilt by association. As best as can be determined, the infrared properties of BL Lac objects are identical to the optical properties of these objects, and since the optical emission is probably due to incoherent electron synchrotron emission, the same must be true for the infrared emission.

Figure 1 shows the energy distribution of the BL Lac object AO $0235+164$ at maximum light in its outburst of 1976 while Figure 6 illustrates the energy distribution of several BL Lac objects extending from the radio through the optical. All these objects show an energy distribution that is relatively flat through the radio, with a steepening to shorter infrared and optical wavelengths. Such behavior is expected of a synchrotron source which has multiple components at longer wavelengths, becomes optically thin to synchrotron self absorption at $\sim 1 \mathrm{~mm}$, and reverts there to the classical power law flux distribution

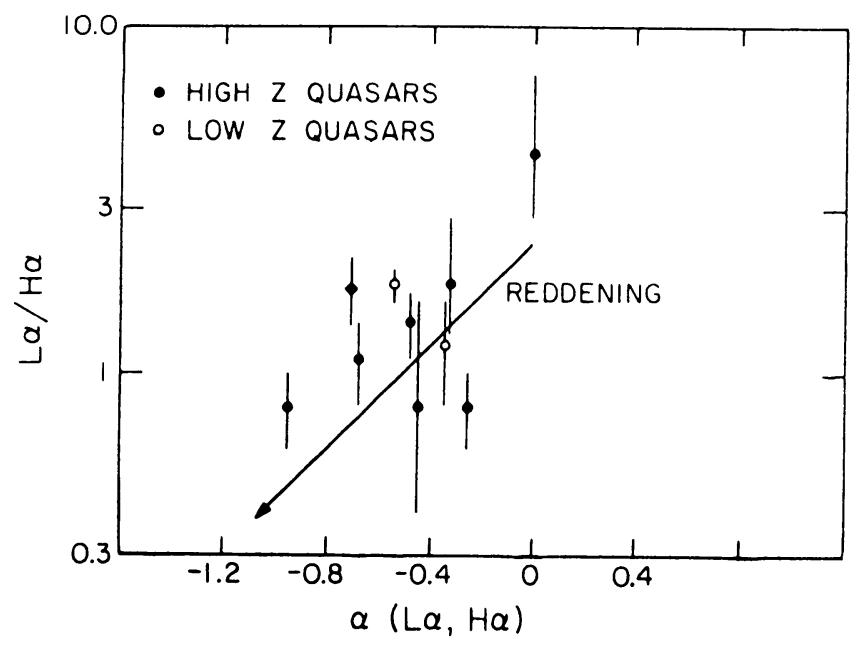

Figure 10. The $\mathrm{L} \alpha / \mathrm{H} \alpha$ line ratio plotted vs. the continuum spectral index $\alpha$ for quasars. The open circles are for the low redshift quasars $3 \mathrm{C} 273$ and PG0026+129. The diamond is a high redshift quasar from Puetter, et al. (1980). All other data are from Soifer, et al (1980a). The arrow indicates the effect increasing external reddening would have on both the lines and continuum, starting with a specific intrinsic spectrum. 
of an optically thin synchrotron source. The steepening at near infrared and optical wavelengths is taken as indicative of increasingly rapid energy loss in the highest energy electrons.

Related to the energy distributions are the polarization properties of these objects. Although the polarization properties of BL Lac objects are well studied optically (see Angel and Stockman 1980 for a review) there has been comparatively little work on the polarization properties of the infrared emission in BL Lac objects. Where studies are available (e.g. Knacke, Capps, and Johns 1976, 1979, Rieke, et al. 1977, Moore, et al. 1980, and Puschell and Stein, 1980), it is generally found that the strength and position angle of the infrared radiation agrees with that found visually, although there are notable exceptions.

By far the most stringent arguments against thermal emission from BL Lac objects comes from their observed variability. Figure 12, from Rieke and Lebofsky (1979a), shows the variation at $2 \mu \mathrm{m}$ of the $\mathrm{B} 1$ Lac object $\mathrm{A} 00235+164$. If this object is at a distance inferred from observed absorption line red-shifts, the observed variations are many times faster than allowed for emission from a thermal source. Again the similarity in the optical and infrared variability of $\mathrm{A} 00235+164$ leads to the almost inescapable conclusion that the same

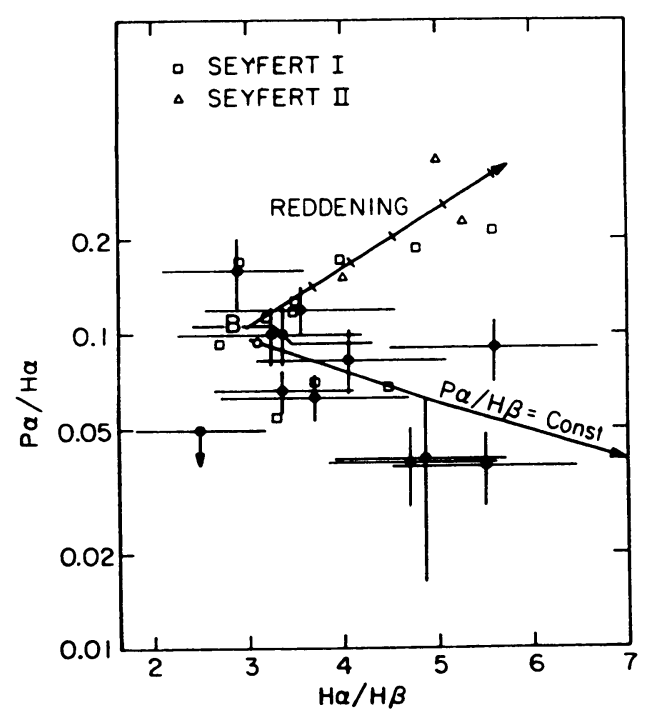

Figure 11. Same as Figure 9 with the addition of observations of Seyfert galaxies from Soifer, et al. (1980b). The tics along the reddening line show successive amounts of $\Delta E(B-V)=0.1$. Note all the Seyfert II nuclei and many of the Seyfert I nuclei follow this track. 


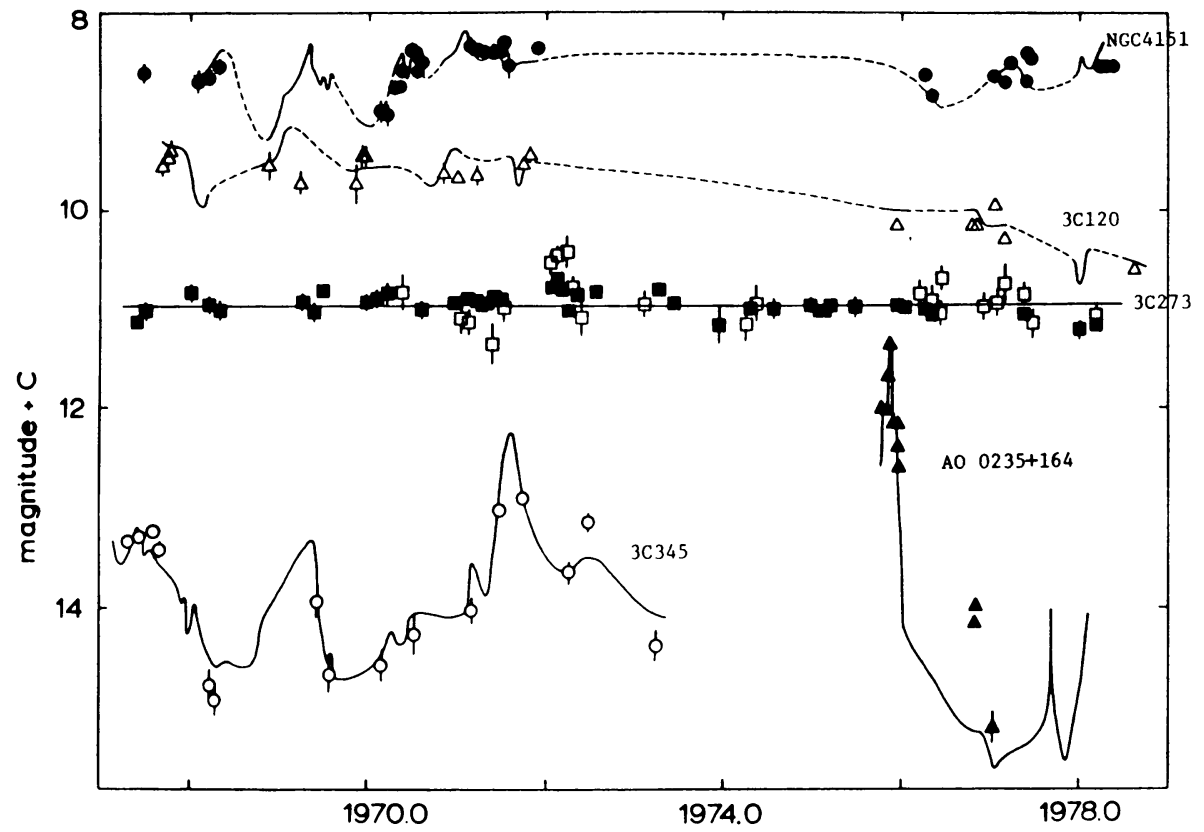

Figure 12. The variability at $2 \mu \mathrm{m}$ (and $10 \mu \mathrm{m}$ open boxes for 3C273) of a variety of extragalactic sources (from Rieke and Lebofsky, 1979a).

emission mechanism is at work in both cases. Figure 13 shows Caltech observations of BL Lac itself and 0J287, measured over the same time scales. There is no evidence for variability in the infrared colors, and therefore the spectral energy distributions, as the continua vary by several magnitudes. This is in contrast to the variable energy distributions seen in the objects discovered by Rieke, et al. (Figure 8).

While it is true that the observed variability in objects such as $0235+164$ (Rieke, et al. 1976) and $1308+326$ (Moore, et al. 1980) stretches the incoherent synchrotron models to their limits and may in fact require non-isotropic emission processes (e.g. Blandford and Rees, 1978) the evidence seems overwhelming that the same emission process is powering the optical, infrared, and radio emission in these objects.

While type 2 Seyfert galaxies and BL Lac objects present the two extreme types of luminous infrared emitters, and appear to present clear-cut evidence for two distinct infrared emission mechanisms, the situation for type 1 Seyfert galaxies and quasars is by no means as clear-cut.

In the case of the quasars, a distinction can be made in the 


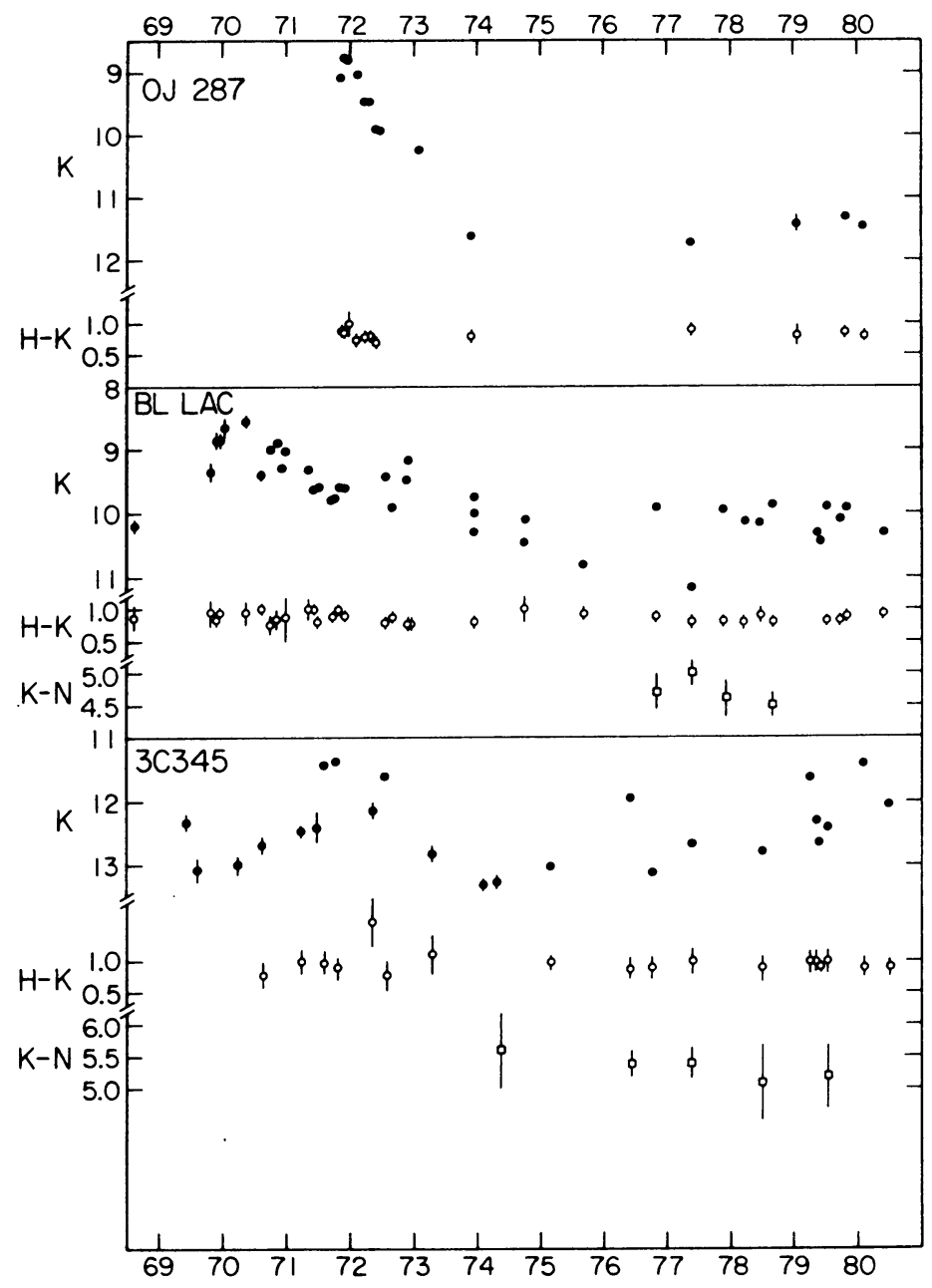

Figure 13. The time history of the $\mathrm{K}$ Magnitude and the $\mathrm{H}-\mathrm{K}$ color for the BL Lac objects BL Lac and 0J287, and the optically violently variable quasar 3C345 (Neugebauer, et al., unpublished). For BL Lac and $3 \mathrm{C} 345$ the $\mathrm{K}-\mathrm{N}$ color is also shown, where it was observed.

class of quasars known as optically violently variable. The members of this class which have been extensively observed at infrared wavelengths, 3C279, 3C 345, 3C446, and 3C454.3, all show a high degree of polarization and variability at $2 \mu \mathrm{m}$ of greater than 1 magnitude over time scales of several years (Neugebauer, et al. 1979); Figure 13 illustrates the $2 \mu \mathrm{m}$ flux measured by the Caltech group of the quasar 3C 345. The range in observed flux is nearly as large as for the BL Lac objects OJ 287 and BL Lac, with similar variability time scales. Angel and Stockman (1980) have argued that these violent variable 
quasars also have the "simplest" power law energy distributions among the quasars, show significant polarization and thus present an extension of the BL Lac class of objects. Similar arguments apply here for the efficacy of the incoherent synchrotron mechanism to produce the infrared emission.

The non-violently variable quasars by definition do not show large variability, and do not show a large degree of polarization, either at optical or infrared wavelengths (Stockman and Angel 1978). While the infrared energy distributions of these quasars are more complex than those of the highly variable quasars, and show a decrease in slope between 3 and $10 \mu \mathrm{m}$, the energy distributions still do not bear any strong similarity to those sources that are believed to show a thermal emission spectrum, and are quite similar to the energy distributions of the "simple spectrum" quasars and BL Lac objects. The energy distribution of $3 \mathrm{C} 273$, the best studied quasar at infrared wavelengths, is shown in Figure 1. There is no evidence for any significant excess above an extrapolated power law spectrum $\mathrm{F}_{\mathcal{V}} \alpha \mathcal{V}^{-1}$ between $20 \mu \mathrm{m}$ and $1 \mathrm{~mm}$. Again this argues for the incoherent synchrotron mechanism. Figure 12 shows the time history of the $2 \mu \mathrm{m}$ flux from 3C273. No positive evidence for variability can be seen from these data.

Other observations that have searched for evidence that indicates the association of dust with quasars have been mostly negative. Searches for reddening induced effects on quasar spectra have been negative. Searches of the optical spectra of quasars of moderate to high red-shift have found nearly no evidence for the $2175 \AA$ absorption typical of galactic type dust. Recent studies of the hydrogen lines in quasars (e.g., Soifer, et al. 1980a; Puetter, et al. 1980); (Figure 9), indicate a general weakening of $\mathrm{P} \alpha$ with respect to $\mathrm{H} \alpha$ as the Balmer decrement increases. The arrow in Figure 9 indicated the track that would be followed if the $\mathrm{P} \alpha / \mathrm{H} \alpha$ and $\mathrm{H} \alpha / \mathrm{H} \beta$ ratios were defined by extinction due to dust in the line emitting region. The observations clearly seem inconsistent with the presence of dust either mixed with the line emitting gas or in the line of sight to it. Furthermore, the $\mathrm{P} \alpha / \mathrm{H} \alpha$ ratio is generally less than that expected for a typical H II region (Case $B$ ), again inconsistent with reddening by dust.

The observations of Figure 10 show that the high and low red-shift quasars share the anomalous $\mathrm{L} \alpha / \mathrm{H} \alpha$ line ratio first suggested by Baldwin (1977). Several authors have suggested that this is in fact due to external reddening. The fact that the low and high red-shift quasars share this property forces the reddening to be local to the quasar. There is a marginal correlation of continuum spectral index with $\mathrm{L} \alpha / \mathrm{H} \alpha$ ratio, seen in Figure 10 , that would be consistent with this model, but this is at best a very weak correlation. Furthermore, the lack of a strong infrared excess in $3 \mathrm{C} 273$ as seen in Figure 1 appears to be inconsistent with this result. 


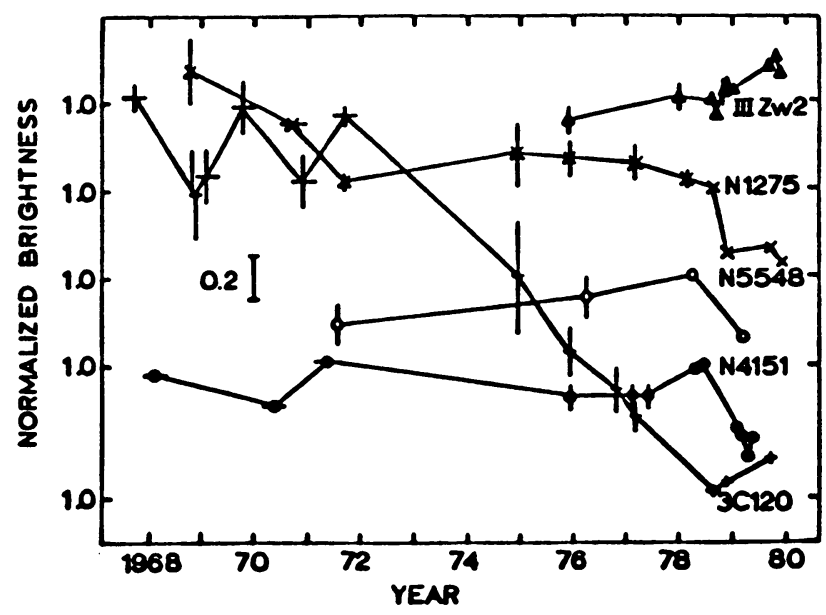

Figure 14. The time history of the infrared flux from Seyfert I galaxies (from Lebofsky and Rieke, 1980).

In contrast to the above arguments, evidence for dust in 3C273 has been given by Allen (1980). The emission is attributed to the $3.3 \mu \mathrm{m}$ emission feature of unknown origin seen from many galactic dustcontaining regions (see e.g. Russell et al. 1977). The detection of this feature in $3 \mathrm{C} 273$ is of low enough signal to noise to warrant confirming observations. If confirmed this would provide strong evidence for some thermal dust emission in quasars. Furthermore, Hyland and Allen (this conference) argue that the energy distribution of quasars steepens into the infrared between 1 and $3 \mu \mathrm{m}$ in a way consistent with a hot dust component.

In summary then, the evidence as it now stands must favor nonthermal emission mechanisms to produce the majority of the infrared flux in quasars, although the evidence is mostly circumstantial, and not as strong as one would like. Furthermore there are data which show that in some quasars dust is in fact present.

The last class of active nuclei that we want to discuss is the type 1 Seyfert galaxies. These galaxies have long been thought to bear significant morphological resemblances to quasars and the energy distribution of the type 1 Seyfert galaxy NCG 4151 is included in Figure 1. This energy distribution is seen in the infrared to resemble more closely that of the quasar 3C273 than that of the Seyfert galaxy NCG 1068. This might be taken as an argument favoring non-thermal emission, and indeed several authors (Stein and Weedman 1976, Neugebauer et al. 1976, McAlary, et al. 1979) have made such arguments. On the other hand, Rieke (1978) has interpreted this distribution as a sum of power law (ultraviolet), stellar, and thermal 
infrared components and has shown that this interpretation, with a significant thermal component, is usually capable of fitting the observations.

Because both interpretations are possible, the energy distributions cannot be decisive evidence in favor of any emission mechanism, and other evidence must be found. Since the polarization in Seyferts is generally weak (Angel and Stockman 1980), this cannot be used as positive evidence for any emission mechanism.

High variability has often been used as an argument in favor of non-thermal emission mechanisms, but in type 1 Seyfert galaxies the observed time scales for infrared variability are compatible with the thermal dust emission mechanism. Recently Rieke and Lebofsky (1979b) and Lebofsky and Rieke (1980) have reported variability of Seyferts that far exceeds the errors in the observations. This variability is shown in Figure 14; unpublished observations at Caltech appear to confirm this variability. Rieke and Lebofsky and Lebofsky and Rieke have argued that the observed variability time scale in type 1 Seyfert galaxies is consistent with thermal emission models. Indeed, they find an increase in the infrared emission from the nucleus of IIIZw2 in late 1979, about two years after the large optical-UV- radio outburst in this galaxy, to be consistent with thermal dust emission at a distance of $2 / 3 \mathrm{pc}$ from the central source.

Spectral features in the infrared emission itself support the model of thermal dust emission providing a significant contribution to the infrared flux in type 1 Seyfert nuclei. Rieke (1976) has reported that the $10 \mu \mathrm{m}$ spectrum of Mk 231 shows absorption characteristic of silicate dust. While this only proves the $10 \mu \mathrm{m}$ source is obscured by dust, it can be taken as evidence for thermal emission at $10 \mu \mathrm{m}$. Recent spectroscopic observations of Mk 231 at $10 \mu \mathrm{m}$ by Jones, et al. (1980) and Gillett (private communication) show a suggestion of an emission band at $11.3 \mu \mathrm{m}$ in the rest frame of $\mathrm{Mk} 231$. If this is confirmed, it would provide direct evidence for thermal emission in this type 1 Seyfert galaxy, since the $11.3 \mu_{\text {im }}$ featuse is identified with thermal dust emission in galactic infrared sources.

More potential evidence for thermal emission in NGC 4151 comes from Cutri and Rudy (1980) who report observations of an emission feature at $3.3 \mu \mathrm{m}$ which they attribute to the feature commonly associated with thermal emission from dust in galactic sources. The most straightforward interpretation of this observation, as thermal emission by material similar to that found in infrared sources in the Galaxy, is made difficult by the absence of a corresponding $11.3 \mu \mathrm{m}$ feature (Jones et al. 1980 Gillett, private communication). If, however, this interpretation is confirmed, it would again provide proof of significant thermal emission in a type 1 Seyfert galaxy.

Indirect evidence that thermal dust emission may be important in Seyfert nuclei comes from spectroscopic observations of the hydrogen 
lines in the spectra of Seyfert galaxies. Figure 11 shows the $\mathrm{P} \alpha / \mathrm{H} \alpha / \mathrm{H} \beta$ ratio for a sample of Seyfert galaxies (Soifer, et al. (1980b). This plot shows that a significant number of the hydrogen line ratios appear to be affected by reddening in the line of sight to the lineemitting regions. This behavior is in fact distinctly different from that of quasars, which show no such effect (see Figure 9).

Thus, in the case of the type 1 Seyfert nuclei, the evidence seems to be increasing that in many instances at least a significant fraction of the emitted infrared radiation is due to thermal dust emission. This is almost certainly a contrast to quasars, where there is no compelling evidence for thermal dust emission contributing predominately to the observed infrared flux.

\section{ACKNOWLEDGEMENTS}

We would like to thank our colleagues who provided preprints of unpublished work. We especially thank A. R. Hyland, Steve Willner, Fred Gillett and Barbara Jones for making available to us and discussing unpublished data. Infrared astronomy at the California Institute of Technology is supported by grants from the NSF and from NASA.

\section{REFERENCES}

Aaronson, M. and Boroson, T.: 1980, Nature 283, p. 746.

Allen, D. A.: 1980 , Nature 284 , p. 323.

Ange1, J. R. P. and Stockman, H. S.: 1980, Ann.Rev.Astron.Astrophys. 18 , in press.

Baldwin, J. A.: 1977, M.N.R.A.S. 178, p. 67P.

Becklin, E. E., Matthews, K., Neugebauer, G., and Wynn-Williams, C. G.: 1973, Astrophys.J. 186, p. L69.

Blandford, R. D. and Rees, M. J.: 1978 in Pittsburgh Conference on

BL Lac Objects, A. M. Wolfe, ed., Univ. of Pittsburgh Press, p. 328.

Cutri, R. and Rudy, R.: 1980, preprint.

Ennis, D. J.: 1980, Ph.D. Thesis, California Institute of Technology, in preparation.

Hildebrand, R. H., Whitcomb, S. E., Winston, R., Stiening, R. F., Harper, D. A., and Moseley, S. H.: 1977, Astrophys.J. 216, p. 698. Jones, B., Merrill, K. M., Stein, W. A., and Willner, S. P.: 1980, private communication.

Jones, T. W., Rudnick, L., Owen, F. N., Puschell, J. J., Ennis, D. J. , \& Werner, M. W.: 1980, Astrophys.J., in press.

Kleinmann, D. E., Gillett, F. C., and Wright, E. L.: 1976, Astrophys.J. 208, p. 42 .

Knacke, R. F., Capps, R. W., and Johns, M.: 1976, Astrophys.J. 210, p. L69.

Knacke, R. F., Capps, R. W., Johns, M.: 1979, Nature 280, p. 215.

Lebofsky, M. J., and Rieke, G. H.: 1980, Nature 284, p. 410. 
McAlary, C. W., McLaren, R. A., and Crabtree, D. R.: 1979, Astrophys.J. 234, p. 471.

Moore, R. L., Ange1, J. R. P., Rieke, G. H., Lebofsky, M. J., Wisniewski, W. Z., Mufson, S. L., Vrba, R. J., Miller, H. R., McGimsey, B. Q., Williamon, R. M.: 1980, preprint.

Neugebauer, G., Morton, D., Oke, J. B., Becklin, E. E., Daltabuit, E., Matthews, K., Persson, S. E., Smith, A. M., Soifer, B. T.,

Torres-Peimbert, S., Wynn-Williams, C. G.: 1980, Astrophys.J. 238 , p. 502 .

Neugebauer, G., Becklin, E. E., Oke, J. B., and Searle, L.: 1976, Astrophys.J. 205, p. 29.

Neugebauer, G., Oke, J. B., Becklin, E. E., and Matthews, K.: 1979, Astrophys.J. 230, p. 79.

0'Dell, S. L.: 1978, in Active Galactic Nuclei, Hazard, C. and Mitton, S., eds., Cambridge Univ. Press, p. 95.

O'De11, S. L., Pusche11, J. J., Stein, W. A., Owen, F., Porcas, R. W., Mufson, S., Moffett, T. J., and Ulrich, M-H.: 1978, Astrophys.J. 224, p. 22 .

Puetter, R. C., Smith, H. E., Willner, S. P., and Pipher, J. L.: 1980, Astrophys.J., in press.

Pusche11, J. J. and Stein, W. A.: 1980, preprint.

Rieke, G. H.: 1976, Astrophys.J. 210, p. L5.

Rieke, G. H.: 1978, Astrophys.J. 226, p. 550 .

Rieke, G. H., Grasdalen, G. L., Kinman, T. D., Hintzen, P., Wills, B. J., and Wills, D.: 1976, Nature 260, p. 754.

Rieke, G. H. and Lebofsky, M. J.: 1979a, Ann.Rev.Astron.Astrophys. 17, p. 477.

Rieke, G. H. and Lebofsky, M. J.: 1979b, Astrophys.J. 227, p. 710.

Rieke, G. H., Lebofsky, M. J., Kemp, J. C., Coyne, G. V., Tapia, S. : 1977, Astropys.J. 218, p. L37.

Rieke, G. H., Lebofsky, M. J., and Kinman, T. D. : 1979, Astrophys.J. 232 , p. L151.

Russe11, R. W., Soifer, B. T., and Merri11, K. M.: 1977, Astrophys.J. 213, p. 66 .

Shields, G. A. and Oke, J. B.: 1975, Astrophys.J. 197, p. 5.

Soifer, B. T., Neugebauer, G., Becklin, E. E., Matthews, K., Oke, J. B., and Malkan, M.: 1980b, in preparation.

Soifer, B. T., Neugebauer, G., Oke, J. B., and Matthews, K.: 1980a, Astrophys.J., in press.

Stein, W. A. and Weedman, D. W.: 1976, Astrophys.J. 205, p. 44.

Stockman, H. S. and Ange1, J. R. P.: 1978, Astrophys.J. 220, p. L67.

Telesco, C. M., Becklin, E. E., and Wynn-Williams, C. G.: 1980, Astrophys.J. (Letters), in press.

Wampler, E. J. : 1968, Astrophys.J. 154, p. L53.

Wampler, E. J.: 1971, Astrophys.J. 164, p. 1. 
DISCUSSION FOLLOWING PAPER DELIVERED BY B. T. SOIFER

STEIN: Optical spectropolarization studies of the nucleus of NGC 4151 indicate the presence of dust. However the amount of dust is much less than in the case of NGC 1068, for example, from various points of view.

THOMPSON: To your 1ist of properties that indicate dust in NGC 1068 you should add what Steve Beckwith stated in his review, that the ratio of $2 \mu \mathrm{m} \mathrm{H}_{2}$ emission-line luminosity to the far infrared luminosity was the same in Orion as in NGC 1068. NGC 4151, which probably has less dust than NGC 1068, does not show that line.

RIEKE: The tendency for the QSO spectral indices between optical and infrared to lie near -1 is at least partly due to selection. When indices are measured for flat-spectrum radio sources selected for radio brightness (irrespective of optical-infrared brightness), the indices have a broad distribution from 0 to -3 .

SOIFER: We may be seeing that effect in the histogram I showed [Figure 5], where there is some suggestion of a tendency for the radioselected quasars to be slightly redder on average than the opticallyselected ones.

BECKLIN: Has anyone measured at $\lambda>2.2 \mu \mathrm{m}$ the steep blank-field QSOs first measured at $2.2 \mu \mathrm{m}$ by Rieke et al.?

SOIFER: Beichman has attempted to measure several of them, but has not detected any.

LEBOFSKY: One of the very red QSOs has been measured at wavelengths longer than $2 \mu \mathrm{m} ; 1413+135$ has been measured from $3.5 \mu \mathrm{m}$ to $10 \mu \mathrm{m}$, and the spectrum at $3.5 \mu \mathrm{m}$ already falls significantly below a powerlaw extrapolated from the 1-2.2 $\mu \mathrm{m}$ data.

BECKLIN: You should not leave the impression that all of the infrared emission in Seyfert Galaxies is thermal radiation from dust, especially at $\lambda<10 \mu \mathrm{m}$. A significant infrared nonthermal source is not ruled out.

SOIFER: At least a majority of the infrared flux is probably thermal, although there is clearly a non-thermal source as well, especially in the optical and $U-V$.

RICKARD: There was once a problem with the steepness of the submillimeter spectrum of NGC 1068, which because of strong upper limits at $1 \mathrm{~mm}$ was too steep to be thermal emission.

SOIFER: To the best of my understanding the infrared spectrum is completely consistent with thermal emission. 
TOVMASSIAN: You presented convincing evidence of nonthermal emission of BL Lac objects and quasars. Are there any explanations of the origin of synchrotron emission in these objects? Is it not the explosion in the nucleus which causes their radio emission?

SOIFER: I refer this question to Wayne Stein.

STEIN: I would rather not be burdened with the task of explaining the ultimate source of energy at this time. 